\title{
Recent Progress of Diamond Device toward Power Application
}

\author{
Shinichi Shikata ${ }^{1, a}$, Kazuhiro Ikeda ${ }^{1}$, Ramanujam Kumaresan ${ }^{1}$, \\ Hitoshi Umezawa ${ }^{1}$ and Natsuo Tatsumi ${ }^{2}$
}

1 Diamond Research Center, National Institute of Advanced Industrial Science and Technology, (AIST) Tsukuba, 305-8568, Japan

${ }^{2}$ Sumitomo Electric Industries Co. Ltd., Hyogo 664-0016, Japan

as-shikata@aist.go.jp

Keywords: diamond, SBD, high temperature, defect, FOM

\begin{abstract}
Diamond is nominated as a material candidate for future high power device due to its superior material properties and resulting very high FOM. In this paper, our recent progresses and the expected possibilities of diamond for power electronics applications are introduced as short review. Firstly for the epitaxial growth, by adopting step-flow epitaxial growth by off- angle substrate with optimized growth conditions, we have succeeded in reducing these killer defects almost six orders from $106 \mathrm{~cm}-2$ to almost $100 \mathrm{~cm}-2$ levels. For the substrate, our recently developed technology to fabricate diamond plates from bulk, $12 \times 13 \mathrm{~mm} 2$ size are available to use, that can avoid fabrication difficulties with small size substrate. Secondly for the device, primitive studies showed possibly for the advantage of diamond such as low reverse leakage current, high temperature and high current density operation.
\end{abstract}

\section{Introduction}

Diamond is nominated as a material candidate for future high power device due to its superior material properties and very high FOM (Figure of Merit) [1, 2]. However, the research activities on diamond device have been focused on lateral structure FET using hydrogen terminated 2 dimension hall gas (DHG) channel, looking for high frequency characteristics, and few activities are found for power device using drift epitaxial layer. But for these several years, many progresses have been reported for both single crystal wafer and devices such as shottky barrier diode in several research institutes. In this paper, our recent progresses and the expected possibilities of diamond for power electronics applications are introduced as short review.

\section{Materials}

The major topic of our current research of diamond power related device is to verify the advantage of diamond by fabricating schottky barrier diode (SBD) using p- drift layer / p+ / insulating substrate structure. For the substrates, only the limited size, approximately $3 \times 3$ to $4 \times 4 \mathrm{~mm}^{2}$ high pressure and high temperature (HPHT) single crystal were commercially available for long time. These tiny size substrates prevent various kind of fabrication process techniques associated with so-called "wafer edge exclusion" issues. However, due to the very recent progress of making large size insulating diamond wafer by microwave CVD, size is increasing and currently $12 \times 12 \mathrm{~mm}^{2}$ substrates are available [3]. Another bottleneck of fabricating wafers is the "slice" technology of diamond, however the conventional laser cutting technique can not be applied to the size larger than $10 \times 10 \mathrm{~mm}^{2}$. This problem has been solved by the sophisticated technology of "slice free" direct wafer lift-off technique [4] using ion implantation, post-growth and wet etching. One of the advantages of using the substrates fabricated by this technology is that we can have the substrate "defect" data, prior to the fabrication of devices by "sister" wafer analysis. 
Next concern for diamond material is that whether the current defect plays a fatal roll for the device performance or not. We have observed excellent rectifying properties of SBD up to 12 orders of magnitude for the defect free area, and the epitaxial crystal defect named as "nonepitaxial crystallites" ( NC) plays an important roll and gives fatal effect on diode characteristics bringing ohmic like leakage current [5]. NC is defined as "killer defect" for diamond, very much like the "Micro pipe" defect for SiC. By adopting step-flow epitaxial growth technique by off angle substrate with optimized growth conditions of high plasma intensity, we have succeeded in reducing these killer defects almost six orders from $10^{6} \mathrm{~cm}^{-2}$ to almost $10^{0} \mathrm{~cm}^{-2}$ level [6]. It is presumably due to the step flow like growth of diamond. Another types of defects are under the investigation using X-ray topography with etch pit formation by using $\mathrm{H}_{2}+\mathrm{CO}_{2}$ dry etching technique [7]. As shown in Fig.1, two types of defects appeared after the dry etching. First type is large size deep pit originated from the epi-layer interface and seems like screw type dislocation, and the second type is normal pit of small size with shallow depth. Density of the deep pits is order of $10^{4} \mathrm{~cm}^{-2}$ and that of the normal type pit is order of $10^{5} \mathrm{~cm}^{-2}$. Further analysis of the defects is under the investigation using X-ray topography image. Influences of these pits to characteristics of SBD have been investigated. The reverse leakage characteristics dependence of the defect density are investigated on the SBD reverse leakage current. The reverse voltage of SBD which gives the leakage current of $1 \mu \mathrm{A} / \mathrm{cm}^{2}$ and $1 \mathrm{~mA} / \mathrm{cm}^{2}$ are plotted in Fig. 2 for many devices in function of the deep pits numbers in schottky contact area. As can be seen from the figure, it is clear that deep pits numbers relates to the reverse leakage. Further studies on defects including classification and using various kind of analysis will be required.

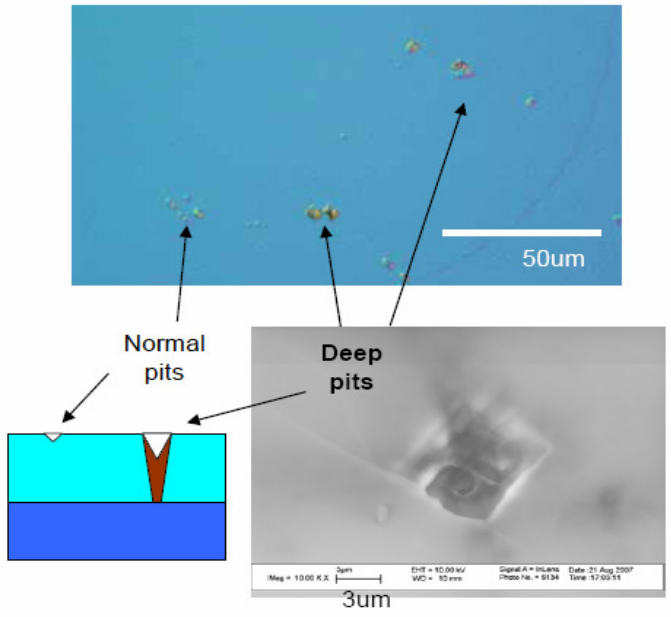

Fig.1 The images of defects after the dry etching.

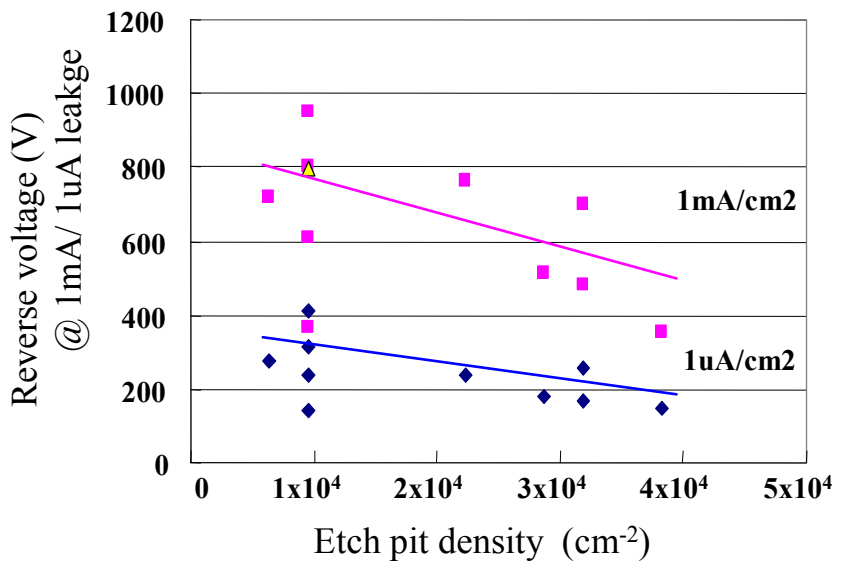

Fig.2 Influence of deep pit density to SBD characteristics.

\section{SBD Device}

Most of the SBDs have been fabricated by "pseudo- vertical" structure that utilizes $p$-drift layer / $\mathrm{p}^{+}$ high doped layer on insulating substrate; that ohmic contact is fabricated laterally on $\mathrm{p}+$ after removing p-. With this structure, high performance SBD can be made as indicated above. The mechanism of the reverse leakage current has been analysed and found that it is in good agreement with the tunnelling model described by thermionic-field emission (TFE) rather than the conventionally used barrier-lowering model [8]. One of the techniques for Schottky contact formation is surface oxidization of diamond to give higher barrier height that results in low reverse leakage current $[9,10]$, and the leakage current of diamond SBDs is observed as low as $10^{-7} \mathrm{~A} / \mathrm{cm}^{2}$ 
(measurement limit) at $1.5 \mathrm{MV} / \mathrm{cm}$, even at the high temperature $(440 \mathrm{~K})$. Breakdown voltage of the fabricated device was $1.6 \mathrm{KV}$ [11]. Currently, the improved growth technique associated with surface oxidation technique resulted in better material characteristics, such as high breakdown field up to $3.1 \mathrm{MV} / \mathrm{cm}$ [9] that has exceeded former reported value of $2.2 \mathrm{MV} / \mathrm{cm}$ [12]. However, the device size is still limited to small size such as $100 \mu \mathrm{m}$ diameter, due to the defects. The typical diode forward characteristics are shown in Fig.3 varying the measurement temperature. And in Fig.4, the reverse characteristics are shown. Diode shows good rectifying characteristics over 8 orders with small reverse leakage current. For the temperature characteristics, hall density of diamond is still in the freeze out range in these temperatures, carrier increases with increasing temperature and exhibit high current density at high temperature.

The passivation technique is also investigated by both numerical simulation and experiments, and it is shown that $\mathrm{Al}_{2} \mathrm{O} 3$ is suitable for the passivation insulator for diamond as field plate[13]. One of the hopeful advantages of diamond is on high temperature operation of the device utilizing high carrier generation of diamond at high temperature, capable of cooling system free device unit. The high temperature operation of SBDs are investigated at 400,600, and 800C, and observed good rectifying characteristics[14]. Further more, the static burn-in testing of Mo and Ru Schottky contact was also carried out at 400C and observed no change of shottky characteristics at all for 1500h[15]. This thermal stability results indicate high temperature operation is possible for diamond. The "vertical" structure SBD was also fabricated by removing the insulating substrate by using ICP dry etching process, and high current density $\left(2980 \mathrm{~A} / \mathrm{cm}^{2}\right)$ and low RonS $\left(1.8 \mathrm{~m} \Omega \mathrm{cm}^{2}\right)$ device have been fabricated[16].

By the primitive studies of Diamond devices looking for future high power applications, some advantages have been highlighted such as; low leakage current, high temperature operation and high current density operation. Associated with the new technology of manufacturing large size substrates, research activities are expected to be enhanced to verify the advantages clearly in near future.

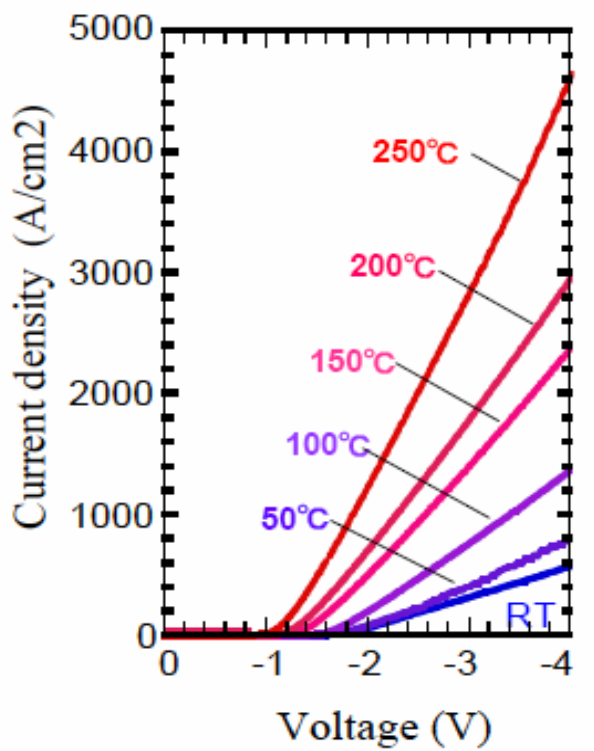

Fig.3 Temperature dependence of forward characteristics of SBD

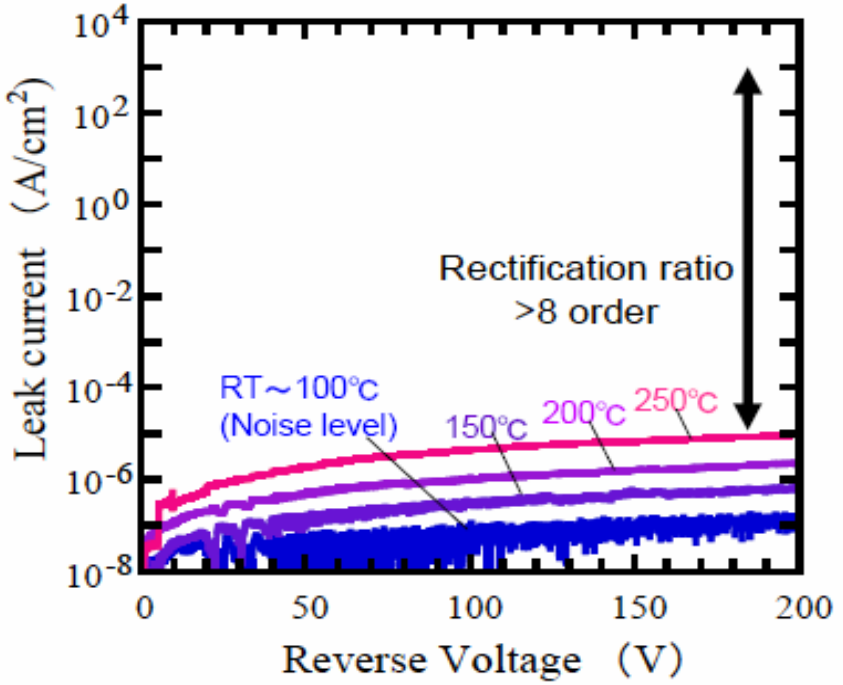

Fig.4 Temperature dependence of reverse characteristics of SBD 


\section{Summary}

For the substrates, recent progress of making large size insulating diamond wafer by microwave CVD, size is increasing and currently $12 \times 12 \mathrm{~mm}^{2}$ substrates are available. Also for the "wafer" fabrication, we are now free from bottleneck of cutting diamond, by the sophisticated technology of "slice free" direct wafer lift-off technique. For the epitaxial film growth, we found that "killer defect" for diamond can be dramatically reduced by adopting step-flow epitaxial growth technique by off angle substrate with optimized growth conditions of high plasma intensity. Other types of defects are under the investigation, and current knowledge is the deep pits which can be found by dry etching seems to affect leakage current. Further studies on defects including classification and using various kind of analysis will be required.

For the device, SBD have been investigated by "pseudo- vertical" structure that utilizes p-drift layer / $p+$ high doped layer on insulating substrate. It is known that very good rectification can be obtained for defect free area with the small reverse leakage determined by thermionic-field emission. By the surface oxidization of diamond to give higher barrier height that results in low reverse leakage current as low as $10^{-7} \mathrm{~A} / \mathrm{cm}^{2}$ at $1.5 \mathrm{MV} / \mathrm{cm}$. Current maximum breakdown field is obtained up to $3.1 \mathrm{MV} / \mathrm{cm}$. For the high temperature operation, high current density can be obtained at high temperature. Newly developed shottky metal by Ru showed high stability at 400C for $1500 \mathrm{~h}$, which support the high temperature operation.

\section{References}

[1] B. J. Baliga: J. Appl. Phys. Vol. 53 (1982), p. 1759

[2] A. Q. Huang: IEEE Electron Dev. Lett. Vol. 25 (2004), p. 298

[3] Y. Mokuno, A. Chayahara, H. Yamada and N. Tsubouchi: ICSCRM 2007, Mater. Sci. Forum, in press (2008)

[4] Y. Mokuno, A. Chayahara and H. Yamada: Diamond and Related Materials 17,415 (2008)

[5] H. Umezawa, N. Tokuda et al.: Diamond and Related Materials Vol. 15 (2006), p. 1949

[6] N. Tatsumi, H. Umezawa, and S. Shikata: ICSCRM 2007, Mater. Sci. Forum, in press (2008)

[7] N. Tatsumi, H. Umezawa et al.: $55^{\text {th }}$ Conf. Jap. Soc. Appl. Phys. (2008), p. 27 p-K-10

[8] H. Umezawa, T. Saito, N. Tokuda, M. Ogura, S. Ri, H. Yoshikawa, and S. Shikata: App. Phys. Lett. Vol. 90 (2007), p. 073506

[9] H. Umezawa, K. Ikeda, N. Tatsumi, and S. Shikata: ICSCRM 2007, Mater. Sci. Forum, in press (2008)

[10]H. Umezawa, K. Shibata, K. Ikeda, N. Tatsumiand S. Shikata: submitted to IEEE Electr. Dev. Lett. (2008)

[11] R. Kumaresan, H. Umezawa, K.Ikeda et al.: Int. Conf. New Dia. Nano Carbons, P2-147 (2008).

[12] S. J. Rashid, L. Coulbeck, A. Tajani, G. A. J.Amaratunga, P. Taylor, M. Dixon, and J. Isberg: Proc. Int. Symp. Power Semiconductor Devices (ISPSD), (2005), p. 315

[13] K. Ikeda, H. Umezawa and S. Shikata, Diamond \& Related Materials Vol. 17 (2008), p. 809

[14] H. Umezawa, K. Shibata, N. Tatsumi et al: $20^{\text {th }}$ Diamond Symposium, Japan (2006), p. 256

[15] K. Ikeda, H. Umezawa, R. Kumaresan and S. Shikata: ICSCRM 2007, Mater. Sci. Forum, in press (2008)

[16] R. Kumaresan, H. Umezawa et al.: $55^{\text {th }}$ Conf. Jap. Soc. Appl. Phys. (2008), p. 27 p-K-11 